UNDERGRADUATE RESEARCH IN NATURAL AND CLINICAL SCIENCE AND TECHNOLOGY (URNCST) JOURNAL Read more URNCST Journal articles and submit your own today at: https://www.urncst.com

\title{
ALSTaR Cells - Novel Stem-cell-based Therapy for Amyotrophic Lateral Sclerosis: A Research Protocol
}

Niharikaa Aiyar, HBSc [1,4,5], Maryam Dadabhoy, HBSc Student [2], Nitya Gulati, HBSc $[1,3]^{*}$

[1] Department of Human Biology, University of Toronto, Toronto, Ontario, Canada, M5S 3J6 [2] Department of Psychology, University of Toronto, Scarborough, Ontario, Canada M1C 1A4 [3] Department of Molecular Genetics, University of Toronto, Toronto, Ontario, Canada M5S 1A8

[4] Department of Cell and Systems Biology, University of Toronto, Toronto, Ontario, Canada, M5S 3G5

[5] Department of Physiology, University of Toronto, Toronto, Ontario, Canada, M5S 1A8

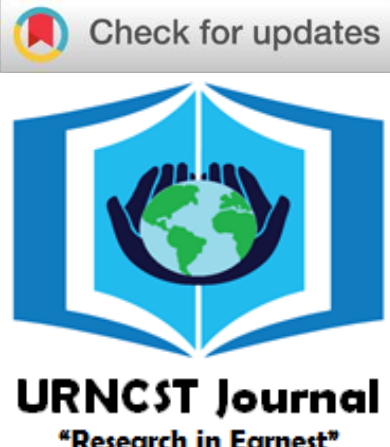

*Corresponding Author: nitya.gulati@ mail.utoronto.ca

\begin{abstract}
Introduction: Amyotrophic Lateral Sclerosis (ALS) is a progressive neurodegenerative disease that results in the loss of motor neurons leading to limb paralysis and eventual death. Stem cell transplantation can be used to replenish the atrophied motor neurons and slow the progression of the disease, while the use of biomaterials, genetic engineering, and nanoparticles can reduce the hostility of the microenvironment. Thus, we propose a novel combinatorial therapeutic approach termed Amyotrophic Lateral Sclerosis Therapeutic and Regenerative (ALSTaR) cell treatment.

Methods: This strategy will employ miRNA-124 and chitosan polyplex biomaterial to enhance engraftment of neural progenitor cells (NPCs), which will be engineered to overexpress an autophagy-regulatory gene, TFEB, and secrete an autophagy-inducing drug, trehalose. We will bioengineer an organoid model of the ventral column of the spinal cord which will be used for extensive in vitro characterization of ALSTaR cells through single-slice electrophysiology and immunocytochemistry. Using the SOD $1_{G 93 A}$ mutant mouse model, weekly behavioural assessments of motor recovery will be conducted until the humane endpoint; thereafter extensive immunohistochemical and protein quantification analyses will be performed to determine treatment efficacy in vivo.

Results: ALSTaR cell treatment should result in motor functional recovery and lifespan elongation through several cellular and molecular mechanisms, including decreased neuroinflammation, upregulation of autophagy, degradation of protein aggregates, enhancement of transplanted cell integration, and regeneration of axons.

Discussion: In vitro characterization of ALSTaR cells in bioengineered spinal cord organoids will reveal stable electrophysiological recordings of motor neurons, higher levels of neuronal differentiation markers, and lower levels of inflammation markers in the ALSTaR group compared to the control group or the groups treated with the biomaterial or the NPCs alone, suggesting neuronal recovery. Behavioural assessments in the ALS mouse model will reveal increased motor coordination, neuromuscular strength, and motor activity in the ALSTaR group compared to other groups, suggesting motor functional recovery. In vivo characterization of ALSTaR cells in the SODIG93A mice will suggest enhanced stem cell integration and recovery of cellular and molecular processes.

Conclusion: With currently no effective treatment for ALS, this novel combinatorial treatment strategy could improve the health-related quality of life of patients suffering from this debilitating disease.
\end{abstract}

Keywords: amyotrophic lateral sclerosis; neurodegeneration; regeneration; NPCs; autophagy; biomaterial; gene overexpression; protein aggregation; spinal cord organoids

\section{Introduction}

Amyotrophic Lateral Sclerosis (ALS) is a fatal, progressive, neurodegenerative disorder of cortical upper motor neurons and brainstem and spinal cord lower motor neurons, with a prevalence rate of 6 in 100,000 and a survival rate of 3-4 years from disease onset $[1,2]$. Clinical manifestations include: skeletal muscle weakness and atrophy, spasticity, dysarthria, dysphagia, cognitive impairment, and eventually respiratory insufficiency and paralysis [2]. Although most patients are diagnosed with unidentifiable causes (sporadic ALS), genetic risk variants have been identified in some patients (familial ALS), particularly mutations in the SODI gene encoding for superoxide dismutase, and the TARDBP gene encoding for TAR DNA-binding protein 43 (TDP-43) [2]. 
UNDERGRADUATE RESEARCH IN NATURAL AND CLINICAL SCIENCE AND TECHNOLOGY (URNCST) JOURNAL Read more URNCST Journal articles and submit your own today at: $\underline{\text { https://www.urncst.com }}$

The pathophysiological mechanisms underlying this devastating disease are copious, thus we will focus our intervention on two crucial pernicious responsesneuroinflammation and protein aggregation. In the diseased microenvironment, microglia change from their neuroprotective phenotype into a toxic one that secretes abnormally high levels of cytokines, leading to cell survival decline [2]. The most pervasive proteinopathies in ALS are the cytoplasmic aggregation of TDP-43 in motor neurons and the downregulation of transcription factor EB (TFEB), which subsequently disrupts the transcriptional regulation of macroautophagy. When the autophagy process is dysregulated, the cell is unable to degrade aggregates of TDP-43 and other proteins [3,4].

Exogenous cell transplantation techniques have extensively been used to provide a promising therapeutic treatment for ALS, as a means of replenishing lost motor neurons, overexpressing depleted genes, and delivering drugs. Stem cells and their progeny provide neuroprotection by interacting with the cytotoxic microenvironment, inhibiting inflammation signalling, and releasing growth factors, thereby promoting axonal growth $[5,6]$. Induced pluripotent stem cells (iPSCs) can be produced from adult tissue, such as skin fibroblasts, and be reprogrammed into autologous pluripotent cells, similar to embryonic stem cells (ESCs) by retroviral transduction encoding transcription factors, such as KLF4, OCT4, SOX2, and c-MYC [7]. Although iPSCs promote functional recovery, their survival rates are poor due to the toxic microenvironment. Hence, combinatorial therapies are investigated, including tissue engineering techniques, which usually combine certain types of cells, bioactive molecules, and biomaterials, like threedimensional (3D) scaffolds [5].

Chitosan is a polyplex system that has been shown to efficiently deliver microRNA-124 (miRNA-124), which targets signal transducer and activator of transcription 3 (STAT3) to decrease inflammatory cytokine levels 6. A polyplex system is ideal, as it is composed of the chitosan polymer that electrostatically interacts with the miRNA-124 nucleic acid to protect it from enzymatic degradation, thereby facilitating its release at the site of inflammation [8]. Chitosan itself can improve the microenvironment of the diseased spinal cord by recovering nerve impulse conduction and reducing the production of reactive oxygen species, so subsequently transplanted stem cells can grow on this scaffold with less inhibition [9]. Additionally, it is biodegradable, biocompatible, and bio-tolerated (i.e., it degrades over time and does not result in inflammatory responses in the body), making it a good choice of biomaterial $[6,10]$.

Previous studies have demonstrated that protein aggregation in ALS can be reduced by promoting autophagy through two mechanisms: (1) direct overexpression of TFEB and (2) delivery of trehalose-a disaccharide drug that upregulates autophagy-regulatory genes, such as Lc3, Becn1, Sqstm1, and Atg5, and transcription factors involved in autophagy activation, such as FOXO1 [3,11,12]. Although trehalose is traditionally delivered intraperitoneally, its bioavailability is low due to the presence of hydrolyzing enzymes in the intestine and kidney $[11,13]$. Thus, packaging trehalose into nanocarriers taken up by stem cells that will subsequently be transplanted into the diseased spinal cord would result in more sustainable and direct drug delivery [14].

Neural organoids are 3D cell culture systems that are developed to model neurodevelopmental and neurodegenerative disorders in humans. This $3 \mathrm{D}$ in vitro system is formed by proliferating, differentiating, migrating, and self-organizing pools of neural progenitor cells (NPCs) and are able to mimic cytoarchitecture, cell type composition, maturity, and functionality of in vivo structures $[15,16]$. We can develop spinal cord organoids to model motor neuron diseases, like ALS.

We propose our novel Amyotrophic Lateral Sclerosis Therapeutic and Regenerative (ALSTaR) cell treatment, which involves the integration of the miRNA-124 and chitosan polyplex biomaterial, with genetically and nanoengineered NPCs that are designed to overexpress TFEB and secrete trehalose. We hypothesize that ALSTaR cell transplantation into the diseased ALS microenvironment of a spinal cord organoid would result in decreased neuroinflammatory responses, upregulated autophagy, decreased toxic protein aggregation, enhanced engraftment of stem cells onto the artificial scaffold, improved axonal regeneration, and subsequently motor functional recovery in the $S O D 1^{G 93 A}$ mutant mouse model. By amalgamating biomaterial, genetic, and nanoparticle technologies, we present a novel combinatorial treatment approach to ALS that utilises various aspects of synthetic biology in an ethical and target-specific manner. Specifically, we opt for the use of iPSCs to avoid the controversial derivation of ESCs from human embryos. Moreover, we precisely target neuroinflammation and protein aggregation to repair the spinal cord microenvironment, thus making it conducive for both endogenous cells that can be rescued and exogenous cells that will be transplanted to replenish lost neurons. The aforementioned studies have demonstrated mild functional recovery and slightly prolonged lifespan with their individualised therapeutic approaches, thus we propose to unite these various molecular applications into one coalesced strategy to treat the multifaceted disease that is ALS $[4,6,11,17]$.

\section{Methods}

Spinal Cord Organoid Models

Adapting protocols in previously published literature, two spinal cord organoid models will be bioengineered using human iPSCs reprogrammed from skin fibroblasts retrieved from either healthy volunteers or ALS-inflicted patients who carry the SOD1 mutation. These iPSCs are expected to recapitulate the neuronal phenotype as seen in previous studies [7]. Firstly, the Wnt pathway will be 
UNDERGRADUATE RESEARCH IN NATURAL AND CLINICAL SCIENCE AND TECHNOLOGY (URNCST) JOURNAL Read more URNCST Journal articles and submit your own today at: https://www.urncst.com

activated in the iPSCs using the lipid molecule, prostaglandin E2, after which they will then be treated with retinoic acid to induce caudalization in three-day-old cultures. A Sonic Hedgehog pathway agonist, Purmorphamine, will be added to cultures from days 10 to 17 to induce ventralization signals. This tissue will then be encapsulated within Matrigel droplets and allowed to expand and grow in a spinning bioreactor with differentiation media [18]. Within a month, the developed spinal cord organoids, of either healthy or ALS phenotypes, will be treated with various combinations of miRNA-124 and chitosan complex biomaterial and the ALSTaR cells (Table 1). Single-slice electrophysiological recordings of motor neurons and immunocytochemical analyses of key neuronal differentiation and ALS-related markers, in the organoids will be compared across conditions (Figure 1).

Table 1. Conditions for in vitro characterization of ALSTaR cells in spinal cord organoids derived from iPSCs from healthy and ALS-inflicted patients. Created on MS Word.

\begin{tabular}{|c|c|c|}
\hline Condition & miRNA-124/Chitosan Complex & iPSC-Derived NPCs \\
\hline Control & - & - \\
\hline Biomaterial & + & - \\
\hline Cells & - & + \\
\hline Biomaterial + Cells & + & + \\
\hline
\end{tabular}

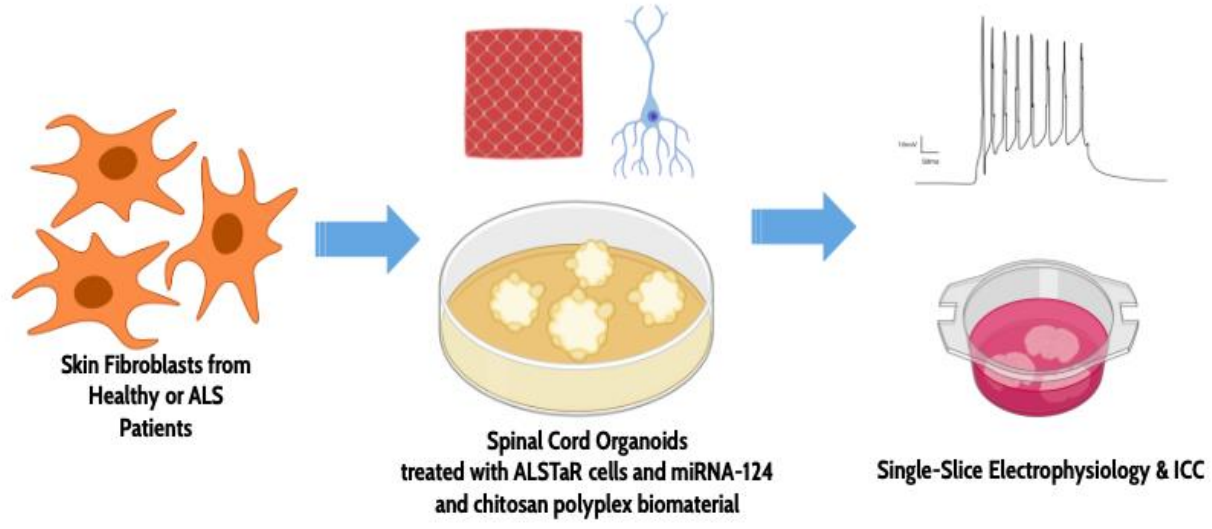

Figure 1. Generation of spinal cord organoid model and experimental procedure for in vitro characterization of ALSTaR cell treatment. Created with BioRender.com.

Animal Model

This study will use 20 wildtype and 80 $S O D 1^{G 93 A}$ mutant mice (purchased from Jackson Laboratory
$\mathrm{USA}^{10}$ ), expressing mutant superoxide dismutase -40 males and 40 females; both sexes to analyse potential sex-based differential disease progression (Table 2).

Table 2. Treatment groups of mice $(n=20,10: 10$ male:female). Created on MS Word.

\begin{tabular}{|c|c|c|c|}
\hline Experimental Group & ALS & $\begin{array}{c}\text { miRNA-124/Chitosan Complex } \\
\text { Injection }\end{array}$ & $\begin{array}{c}\text { iPSC-Derived NPCs } \\
\text { Transplantation }\end{array}$ \\
\hline Control & - & - & - \\
\hline Vehicle + Vehicle & + & - & - \\
\hline Biomaterial + Vehicle & + & + & + \\
\hline Vehicle + Cells & + & + & + \\
\hline Biomaterial + Cells & + & + & + \\
\hline
\end{tabular}




\section{Behavioural Assessments}

Blood samples will be collected from the transgenic mice and checked for the diseased phenotype by conducting reverse transcription polymerase chain reaction (RT-PCR). Thereafter, weekly behavioural and weight assessments will be conducted starting one week post-training until the humane endpoint (i.e., complete paralysis; typical lifespan of 19-23 weeks), including the Rotarod Test, Hanging-Wire
Test, and Treadmill, assessing motor coordination, neuromuscular strength, and motor activity, respectively [17]. All assessments will be performed and scored by two independent, blinded observers to reduce subjective bias. A two-way analysis of variance (ANOVA) test will be used to analyze significant statistical differences across treatment group and sex [17].

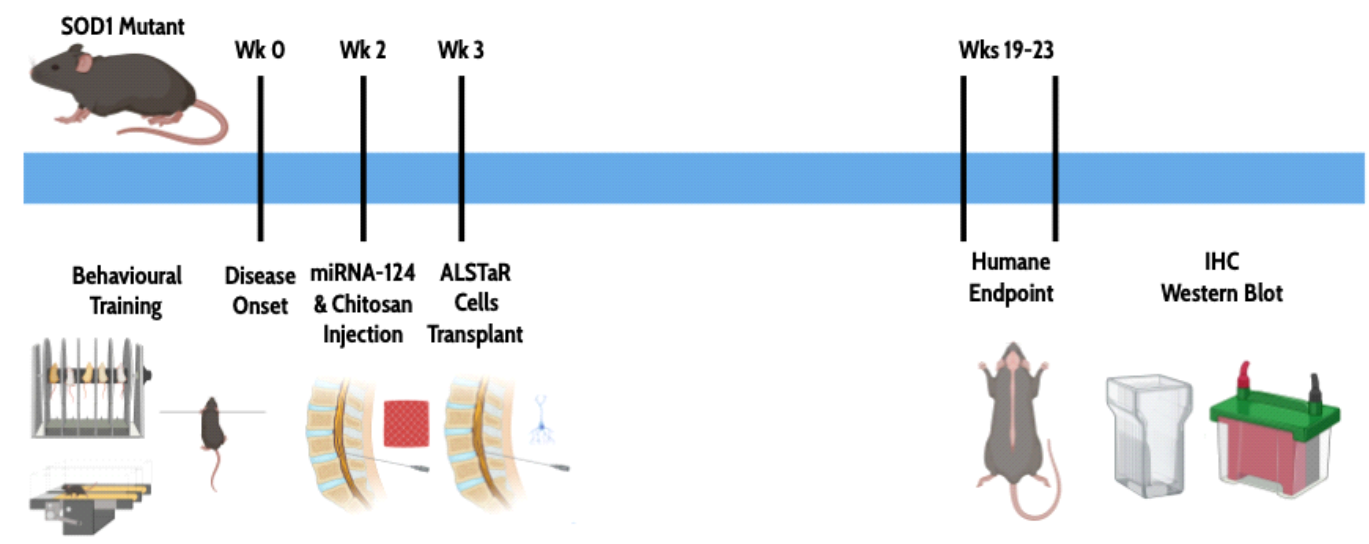

Figure 2. Animal model and experimental timeline for in vivo characterization of ALSTaR cell treatment. Created with BioRender.com.

\section{Cellular and Molecular Analysis}

To create the miRNA-124 and chitosan complex, chitosan, which is $95 \%$ de-acetylated, will be purchased from Heppe Medical Chitosan GMbH (Frankfurt, Germany) and processed to form a $1 \mathrm{mg} / \mathrm{ml}$ solution. The pre-miR ${ }^{\mathrm{TM}}$ miRNA precursor, miR-124, will be diluted in acetate buffer and following this, the chitosan solution will be added to it, resulting in a complex [19]. The miRNA-124 and chitosan complex biomaterial will be injected into the lumbar spinal cord parenchyma two weeks post-diseaseonset. Next, we will overexpress TFEB to counteract autophagy dysfunction and neurodegeneration as a result of ALS. To do this, we will use CRISPR-Cas9 activation methods and transfect NPCs with d-Cas9-VPR with a combination of sgRNAs designed to target the promoter region of the TFEB gene [20]. To increase the transactivation efficiency, we will be using a combination of three sgRNAs and a dual rAAV vector system for dCas9-VPR-mediated TFEB transactivation in NPCs. To further enhance protein aggregate degradation directly in the diseased microenvironment, we will also encapsulate trehalose into a nanocapsule based on methods previously described [21]. These nanocapsules will be delivered into the genetically engineered NPCs by incubating the cells with trehalose-encapsulated nanocapsules at $37^{\circ} \mathrm{C}$ for 40 minutes [21]. These transgenic NPCs labelled with green fluorescent protein (GFP) will be injected in the same region as the biomaterials one week thereafter.

To prevent rejection of human cells, mice will be immunosuppressed by treating them with Cyclosporin A
(CsA) [22]. Although this transplantation process is invasive due to the required laminectomy and local injection into sensitive tissue, we believe parenchymal penetration would be more effective than an intrathecal approach, as it directly targets the toxic microenvironment [23].

The mice will be transcardially perfused at the humane end point, which is marked by complete paralysis, representing end-stage ALS (typical lifespan of 19-23 weeks) [3]. Blood and cerebrospinal fluid (CSF) will be extracted for molecular analysis, and spinal cords and brains will be harvested, cryoprotected, embedded, and cryosectioned for immunohistochemical analysis. Quantification of protein aggregates, namely TDP-43 in the blood and CSF samples, will be performed using a commercially available ELISA kit according to the manufacturer's instructions. In this assay, a biotinylated rabbit polyclonal antibody against TDP-43 will be used [24]. A panel of five cytokines, which include tumor necrosis factor- $\alpha$ (TNF- $\alpha$ ), interleukin-6 (IL-6), interleukin-2 (IL-2), interleukin-10 (IL-10), interferon-gamma (IFN- $\gamma$ )will be analysed using the Bio-Plex Multiplex Immunoassay (BioRad Laboratories). This analytical platform consists of a fluorescent bead-based technology combined with a sandwich immunoassay performed on blood and CSF extracted from the mice [25]. In the brain and spinal cord sections, autophagy will be detected using levels of TFEB and LC3-II protein, which can be detected by immunohistochemical analysis; the antibodies rabbit antiTFEB and rabbit anti-LC3II will be used, respectively. Stem cell integration will be mapped using GFP $[7,11,12]$. 


\section{Results}

We expect to observe increased axonal regeneration, NPC engraftment, and autophagy in our ALS spinal cord organoid upon treatment with ALSTaR cells and chitosan miRNA polyplex biomaterial. These organoids are also expected to show lower levels of neuroinflammation and protein aggregation. In the in vivo study, we expect to observe the longest lifespan and the most improved functional recovery in the mutant mice with miRNA-124 and chitosan polyplex biomaterial microinjection and ALSTaR cell transplantation, with lower quantifications of protein aggregates and higher autophagy levels due to overexpression of TFEB and secretion of trehalose, compared to the other experimental groups. No sexdependent differences are expected to emerge.

\section{Discussion}

This study aims to test the efficacy of the ALSTaR cell treatment in recovering cellular processes damaged by ALS, improving motor function, and elongating lifespan. The results of these experiments will reveal the efficacy of the ALSTaR treatment in an ALS mouse model, which can then be clinically translated to the human population.
Specifically, in vitro characterization of ALSTaR cells in spinal cord organoids will reveal stable electrophysiological recordings of motor neurons, higher levels of neuronal differentiation markers, such as glial fibrillary acidic protein (GFAP) and beta-III tubulin, and lower levels of inflammation markers, such as IL-2, IL-6, IL-10, TNF- $\alpha$, and IFN- $\gamma$ in the ALSTaR group compared to the control group or the groups treated with just the biomaterial or just the NPCs, suggesting neuronal recovery.

After successful results in the spinal cord organoids, in vivo characterization of ALSTaR cells in an ALS mouse model will reveal increased motor coordination, neuromuscular strength, and motor activity, suggesting motor functional recovery in the ALSTaR group compared to other groups. Moreover, molecular analysis of the blood and CSF, and the immunohistochemical analysis performed on the spinal cord and brain sections will reveal lower levels of protein aggregates and cytokines, higher levels of autophagy, and increased stem cell integration in the ALSTaR group, suggesting recovery of cellular and molecular processes that likely underlie the behavioural improvements observed (Figure 3).

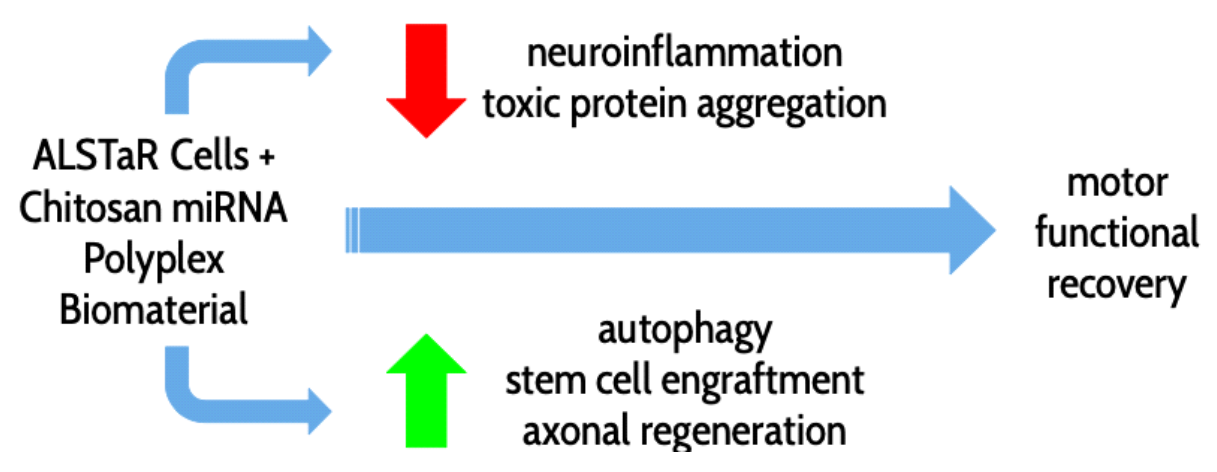

Figure 3. Expected results of the ALSTaR cell treatment in ALS patients. Created with BioRender.com.

Future studies should be conducted to assess the efficacy of the ALSTaR treatment in a diverse cohort of human ALS patients. A major limitation of this study is the use of a mouse model that mimics ALS due to a mutation in the SOD1 gene, which would only represent familial cases of ALS. Most patients are diagnosed with sporadic ALS, thus the observed efficacy of the ALSTaR treatment in this genetic model of ALS may not represent its effects in the variable ALS population.

\section{Conclusions}

This study will explore the ALSTaR cell treatment, which involves the integration of the miRNA-124 and chitosan polyplex biomaterial, with genetically and nanoengineered NPCs that are designed to overexpress TFEB and secrete trehalose, as a novel intervention strategy for
ALS. It is expected that ALSTaR cell transplantation into the

diseased ALS microenvironment of a spinal cord organoid would result in decreased neuroinflammatory responses, upregulated autophagy, decreased toxic protein aggregation, enhanced engraftment of stem cells onto the artificial scaffold, improved axonal regeneration, and subsequently motor functional recovery in the $S O D 1^{G 93 A}$ mutant mouse model. With currently no effective treatment for ALS, a debilitating disease that affects 6 in 100,000 people annually, this novel treatment strategy could improve the health-related quality of life of patients suffering from ALS. Future directions will involve clinical trials in human ALS patients to experimentally test the safety and efficacy of the ALSTaR cell treatment. 
UNDERGRADUATE RESEARCH IN NATURAL AND CLINICAL SCIENCE AND TECHNOLOGY (URNCST) JOURNAL Read more URNCST Journal articles and submit your own today at: https://www.urncst.com

\section{List of Abbreviations Used}

ALS: amyotrophic lateral sclerosis

TARDBP: TAR DNA-binding protein 43

TFEB: transcription factor EB

iPSCs: induced pluripotent stem cells

ESC: embryonic stem cells

3D: three-dimensional

miRNA-124: microRNA-124

NPC: neural progenitor cell

ALSTaR: amyotrophic lateral sclerosis therapeutic and regenerative

RT-PCR: reverse transcription polymerase chain reaction

ANOVA: analysis of variance

GFP: green fluorescent protein

CsA: cyclosporin A

CSF: cerebrospinal fluid

TNF- $\alpha$ : tumor necrosis factor- $\alpha$

IL-6: interleukin-6

IL-2: interleukin-2

IL-10: interleukin-10

IFN- $\gamma$ : interferon- $\gamma$

\section{Conflicts of Interest}

The author(s) declare that they have no conflict of interests.

\section{Ethics Approval and/or Participant Consent}

An informed consent form for obtaining human tissue samples and an Animal Use Protocol (AUP), detailing project rationales, appropriate animal numbers, pre-, intra-, and post-operative monitoring guidelines, and surgical techniques, will be created and approved by the research institution and the Local Animal Care Committee.

\section{Authors' Contributions}

NA: made substantial contributions to the design of the study, drafted and revised the manuscript critically, and gave final approval of the version to be published, and agreed to be accountable for all aspects of the study.

MD: made substantial contributions to the design of the study, drafted and revised the manuscript critically, and gave final approval of the version to be published, and agreed to be accountable for all aspects of the study.

NG: made substantial contributions to the design of the study, drafted and revised the manuscript critically, and gave final approval of the version to be published, and agreed to be accountable for all aspects of the study.

\section{Funding}

This study was not funded.

\section{References}

[1] Talbott E, Malek AM, Lacomis D. The epidemiology of amyotrophic lateral sclerosis. Handbook of Clinical Neurology. 2016 Oct 10;138:225-238. https://doi.org/10.1016/b978-0-12-802973-2.00013-6

[2] Orla H, Chalabi AA, Chio A, Corr EM, Logrocscino G, Robberecht W, et al. Amyotrophic lateral sclerosis. Nature Review Disease Primers. 2017 Oct 05. https://doi.org/10.1038/nrdp.2017.85

[3] Chen Y, Liu H, Guan Y, Wang Q, Zhou F, Jie J et al. The altered autophagy mediated by TFEB in animal and cell models of amyotrophic lateral sclerosis. American Journal of Translational Research. 2015 Sep 15;7(9):1574-1587. Available from: https://www.ncbi.nlm.nih.gov/pmc/articles/PMC46264 $\underline{19 /}$

[4] Silva NA, Sousa N, Reis RL, Salgado AJ. From basics to clinical: A comprehensive review on spinal cord injury. Progress in Neurobiology. 2014;114:25-57. https://doi.org/10.1016/j.pneurobio.2013.11.002

[5] Madhavan L, Ourednik V, Ourednik J. Neural stem/progenitor cells initiate the formation of cellular networks that provide neuroprotection by growth factormodulated antioxidant expression. Stem Cells. 2008;26(1):254-65. https://doi.org/10.1634/stemcells .2007-0221

[6] Louw AM, Kolar MK, Novikova LN, Kingham PJ, Wiberg M, Kjems J, et al. Chitosan polyplex mediated delivery of miRNA-124 reduces activation of microglial cells in vitro and in rat models of spinal cord injury. Nanomedicine: Nanotechnology, Biology and Medicine. 2016;12(3):643-53. https://doi.org/10.1016/j.nano.2015.10.011

[7] Dimos JT, Rodolfa KT, Niakan KK, Weisenthal LM, Mitsumoto H, Chung W, et al. Induced pluripotent stem cells generated from patients with ALS can be differentiated into motor neurons. Science. 2008; 321(5893):1218-21. https://doi.org/10.1016/j.brainres.2014.09.017

[8] Vasile C. Chapter 1 - Polymeric nanomaterials: Recent developments, properties and medical applications. Polymeric Nanomaterials in Nanotherapeutics. 2019;166. https://doi.org/10.1016/B978-0-12-813932-5.00001-7

[9] Cho Y, Shi R, Borgens RB. Chitosan produces potent neuroprotection and physiological recovery following traumatic spinal cord injury. Journal of Experimental Biology. 2010; 213(9):1513-1520. https://doi.org/10.1242/jeb.035162

[10] Jiang T, James R, Kumbar SG, Laurencin CT. Chapter 5-chitosan as a biomaterial: Structure, properties and applications in tissue engineering and drug delivery. Natural and Synthetic Biomedical Polymers. 2014: 91-113. https://doi.org/10.1016/B978-0-12-396983$\underline{5.00005-3}$ 
UNDERGRADUATE RESEARCH IN NATURAL AND CLINICAL SCIENCE AND TECHNOLOGY (URNCST) JOURNAL Read more URNCST Journal articles and submit your own today at: https://www.urncst.com

[11] Castillo K, Nassif M, Valenzuela V, Rojas F, Matus S, Mercado G, et al. Trehalose delays the progression of amyotrophic lateral sclerosis by enhancing autophagy in motoneurons. Autophagy. 2013;9(9):1308-20. https://doi.org/10.4161/auto.25188

[12] Arotcarena M-L, Bourdenx M, Dutheil N, Thiolat M-L, Doudnikoff E, Dovero S, Ballabio A, Fernagut P-O, Meissner WG, Bezard E, Dehay B. Transcription factor EB overexpression prevents neurodegeneration in experimental synucleinopathies. JCI Insight. 2019;4(16):e129719. https://doi.org/10.1172/jci.insight .129719 .

[13] Wada S-I, Kubota Y, Sawa R, Umekita M, Hatano M, Ohba S-I, et al. Novel autophagy inducers lentztrehaloses A, B and C. The Journal of Antibiotics. 2015 Nov;68(8):521-9. https://doi.org/10.1038/ja.2015.23

[14] Fliervoet LA, Mastrobattista E. Drug delivery with living cells. Advanced Drug Delivery Reviews. 2016;106:63-72. https://doi.org/10.1016/j.addr.2016.04.021

[15] Winanto, Khong Z, Hor J, Ng S. Spinal cord organoids add an extra dimension to traditional motor neuron cultures. Neural Regeneration Research. 2019;14:15151516. https://doi.org/10.4103/1673-5374.255966

[16] Hor J, Soh E, Tan L, Lim V, Santosa M, Winanto, Ho B, Fan Y, Soh B, Ng D. Cell cycle inhibitors protect motor neurons in an organoid model of spinal muscular atrophy. Cell Death \& Disease. 2018;9:1100. https://doi.org/10.1038/s41419-018-1081-0

[17] Oliván S, Calvo AC, Rando A, Muñoz MJ, Zaragoza P, Osta R. Comparative study of behavioural tests in the SOD1G93A mouse model of amyotrophic lateral sclerosis. Experimental Animal. 2015;64(2):147-53. https://doi.org/10.1538/expanim.14-0077
[18] Wong CT, Ussyshkin N, Ahmad E, Rai-Bhogal R, Li H, Crawford DA. Prostaglandin E2 promotes neural proliferation and differentiation and regulates $\mathrm{Wnt}$ target gene expression. Journal of Neuroscience Research. 2016 Aug;94(8):759-75. https://doi.org/10.1002/jnr.23759

[19] Louw AM, Kolar MK, Novikova LN, Kingham PJ, Wiberg M, Kjems J, Novikov LN. Chitosan polyplex mediated delivery of miRNA-124 reduces activation of microglial cells in vitro and in rat models of spinal cord injury. Journal of Nanomedicine. 2016 Apr;12(3):643653. https://doi.org/10.1016/j.nano.2015.10.011

[20] Ma, D., Peng, S. \& Xie, Z. Integration and exchange of split dCas9 domains for transcriptional controls in mammalian cells. Nature Communication. 2016;7:13056. https://doi.org/10.1038/ncomms13056

[21]Zhang W, Rong J, Wang Q, He X. The encapsulation and intracellular delivery of trehalose using a thermally responsive nanocapsule. Nanotechnology. $2009 \mathrm{Jul}$ 8;20(27):275101. https://doi.org/10.1088/0957-4484/20/ $27 / 275101$

[22] Antonios JP, Farah GJ, Cleary DR, Martin JR, Ciacci JD, Pham MH. Immunosuppressive mechanisms for stem cell transplant survival in spinal cord injury. Neurosurgical Focus. 2019;46(3):E9. https://doi.org/ $\underline{10.3171 / 2018.12 . F O C U S 18589}$

[23] Pardridge, W.M. Drug transport in the brain via the cerebrospinal fluid. Fluids Barriers of the CNS. 2011;8(7). https://doi.org/10.1186/2045-8118-8-7

[24] Ren Y, Li S, Chen S, Sun X, Yang F, Wang H, Li M, Cui F, Huang X. TDP-43 and Phosphorylated TDP-43 Levels in paired plasma and CSF samples in amyotrophic lateral sclerosis. Frontiers in Neurology. 2021;12:663637. https://doi.org/10.3389/fneur.2021.663637

[25] Tortelli R, Zecca C, Piccininni M, Benmahamed S, Dell'Abate MT, Barulli MR, Capozzo R, Battista P, Logroscino G. Plasma inflammatory cytokines are elevated in ALS. Frontiers Neurology. 2020 Nov $13 ; 11: 552295$. https://doi.org/10.3389/fneur.2020.552295

\section{Article Information}

Managing Editor: Jeremy Y. Ng

Peer Reviewers: Siobhan O’Brien

Article Dates: Received May 24 21; Accepted Aug 19 21; Published Nov 0421

\section{Citation}

Please cite this article as follows:

Aiyar N, Dadabhoy M, Gulati N. ALSTaR Cells - Novel stem-cell-based therapy for amyotrophic lateral sclerosis: A research protocol. URNCST Journal. 2021 Nov 04: 5(11). https://urncst.com/index.php/urncst/article/view/283 DOI Link: https://doi.org/10.26685/urncst.283 
UNDERGRADUATE RESEARCH IN NATURAL AND CLINICAL SCIENCE AND TECHNOLOGY (URNCST) JOURNAL

Read more URNCST Journal articles and submit your own today at: https://www.urncst.com

\section{Copyright}

(C) Niharikaa Aiyar, Maryam Dadabhoy, Nitya Gulati. (2021). Published first in the Undergraduate Research in Natural and Clinical Science and Technology (URNCST) Journal. This is an open access article distributed under the terms of the Creative Commons Attribution License (https://creativecommons.org/licenses/by/4.0/), which permits unrestricted use, distribution, and reproduction in any medium, provided the original work, first published in the Undergraduate Research in Natural and Clinical Science and Technology (URNCST) Journal, is properly cited. The complete bibliographic information, a link to the original publication on http://www.urncst.com, as well as this copyright and license information must be included.

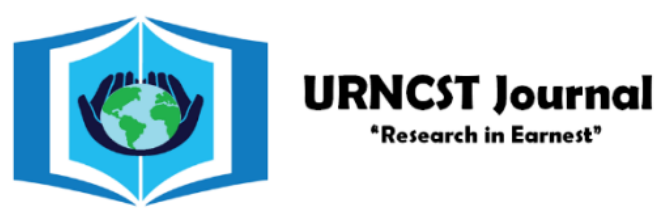

\section{Funded by the Government of Canada}

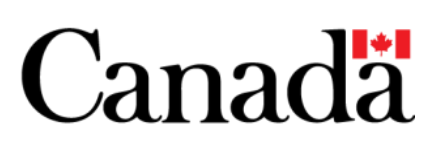

Do you research in earnest? Submit your next undergraduate research article to the URNCST Journal! |Open Access | Peer-Reviewed | Rapid Turnaround Time | International | | Broad and Multidisciplinary | Indexed | Innovative | Social Media Promoted |

Pre-submission inquiries? Send us an email at info@urncst.com | Facebook, Twitter and LinkedIn: @URNCST

Submit YOUR manuscript today at https://www.urncst.com! 